Presented at the Conference on Grazing Incidence Optics for Astronomical and Laboratory

App Iications, San Diego, CA, 8/17-19/87 and subitted to SPIE/Optical Engineering.

BNL-41166

$$
\text { CONF-8708242--2 }
$$

\title{
Mirror and Grating Surface Figure Requirements \\ for Grazing Incidence Synchrotron Radiation Beamlines: \\ Power Loading Efrects
}

\author{
BNL--41166 \\ Sieven L. Hulbert and Sushil Sharma \\ DE88 009836 \\ National Synchrotron Light Source, \\ Brookhaven National Laboratory, Upton, NY 11973
}

\begin{abstract}
At present, grazing incidence mirrors are used almost exclusively as the first optical element in VUV and soft $x$-ray synchrotron radiation beamlines. The performance of these mirrors is determined by thermal and mechanical stress-induced figure errors as well as by figure errors remaining from the grinding and polishing process. With the advent of VUV and soft $\mathrm{x}$-ray undulators and wigglers has come a new set of thermal stress problems related to both the magnitude and the spatial distribution of power from these devices. In many cases the power load on the entrance slits and gratings in these beamlines is no longer negligible.

The dependence of thermally-induced front-end mirror figure errors on various storage ring and insertion device parameters (especially those at the National Synchrotron Light Source) and the effects of these figure errors on two classes of soft $x$-ray beamlines are presented.
\end{abstract}

Subject terms: grazing incidence mirror, surface figure, synchrotron radiation, power loading, thermal stress 


\section{Introduction}

Essentially all synchrotron radiation-based VUV and soft $x$-ray beamlines in use throughout the world today utilize refiection optics for collection and focusing (or defocusing). The transmission and energy resolution of these beamlines depend in varying degrees upon the precise maintenance of the figure of these mirrors. In general, beamlines that utilize higher resolving power $(E / \Delta E)$ monochromators require mirrors with lower figure error in order to achieve high transmission (i.e. high flux throughput).

The demand for higher flux has led to the use of storage ring insertion devices (wigglers and undulators) as very intense sources of synchrotron radiation, independent of the coherent properties of their output. The increased power load and, especially, the increased power density on the optical elements of insertion device beamlines lead to larger thermal gradients in these optical elements and hence to larger internal stresses and larger thermal distortions of their surfaces. As we shall see, these thermally-induced figure errors can reduce the transmission of the beamline, thereby offsetting the benefits of increased flux from the source. In some designs, these figure errors can also reduce the resolving power of the monochromator. It is precisely the desired combination of higher flux and higher energy resolution that will most likely lead to new understanding of the physical processes that occur at the spectroscopically important K-edges of the light elements such as carbon, nitrogen, and oxygen and the L-edges of the third-row elements up to copper (including the $3 \mathrm{~d}$ transition metals), all of which lie in the VUV/soft $x$-ray energy range (20 to $1000 \mathrm{eV}$ ).

Much previous work [1] has been done concerning the best materials, cooling methods, and cooling geometries for specific power loading conditions. Here, we present an integrated set of calculations of the power load distributions of three representative synchrotron sources on a grazing incidence mirror, the resulting thermal gradients and deformations, and the effects of these figure errors on the transmission 
and resolution of two representative soft $x$-ray monochromators.

In sec. 2 we present power load distributions from three synchrotron radiation sources at the National Synchrotron Light Source (NSLS) at Brookhaven National Laboratory incident upon a vertically deflecting mirror at $2^{\circ}$ grazing angle of incidence located $10 \mathrm{~m}$ from the source. In sec. 3 we present the results of thermal and stress analyses of this mirror. In sec. 4 we present ray tracing calculations of the effect of the thermally-induced deformations on the optical performance of the spherical grating monochromator (SGM)[2], one member of a class of soft $x$-ray grazing incidence monochromators that utilize an entrance slit, and the proposed UMO monochromator [3], an entrance-slitless monochromator designed for undulator sources. In sec. 5 we present our conclusions.

\section{Power loads from selected NSLS devices}

To compare the effects of different synchrotron radiation sources on soft $x$-ray beamlines of different optical designs, we choose a fixed mirror size, orientation, material, and cooling geometry. Specifically, we consider a $500 \mathrm{~mm} \times 65 \mathrm{~mm} \times 45$ mm rectangular mirror oriented so that the central ray from the source is incident at a grazing angle of $2^{\circ}$ on the center (the pole) of the $500 \mathrm{~mm} \times 65 \mathrm{~mm}$ face of the mirror in a scattering plane parallel to the long dimension of the mirror. This scattering plane is verical, i.e. perpendicular to the orbital plane of the synchrotron radiation storage ring. The mirror pole is $10 \mathrm{~m}$ from the center of the source, which provides 1.75 mrad vertical acceptance while maintaining high soft $x$-ray reflectivity of the high- $Z$ surface coating (e.g. gold or platinum). The back face edges of the mirror are assumed to be held rigidly by the mirror support, an assumption that will be shown below to be justified for the power loads and mirror materials considered in this paper (the intemal stresses are shown to be very small). In addition, the mirror is assumed to be cooled from the sides by water-cooled copper rails that make perfect thermal 
contact with the mirror (as approximated by a liquid metal interface, for example). We choose $\alpha$-sintered silicon carbide $(\mathrm{SiC})$ as the substrate mirror material due to its high thermal conductivity $(K)$ and low thermal expansion $(\alpha)$. In fact, the thermal deformation figure of merit, $\mathrm{K} / \alpha$, of $\mathrm{SiC}$ is 1.35 times that of copper, 4.5 times that of aluminum, and 12 times that of fused silica [4]. In sec. 3 we demonstrate the effect of changing the mirror substrate material from $\mathrm{SiC}$ to $\mathrm{Al}$, with all other parameters held constant (mirtor geometry, power load magnitude, and power load distribution).

Table I lists the physical parameters that characterize various synchrotron sources along with the total output power and peak power density from these devices. The total power from any synchrotron radiation source varies as the square of the ring energy $E$, as the square of the magnetic field strength $B$, linearly with stored beam current $\mathrm{I}$, and linearly with a characteristic length. For bending magnet sources this characteristic length is the radius of the bend; for insertion devices it is the total length of the device $L=N \lambda_{u}$, where $N$ is the number periods, each of length $\lambda_{u}$. The power density peaks on axis and varies as the fourth power of the ring energy $E$, linearly with the magnetic field strength $B$, linearly with the stored ring current $I$, and, for insertion devices, linearly with the number of periods $\mathbf{N}[5,6]$. In this paper, we consider the NSLS U13 hybrid wiggler/undulator (U13), an NSLS $x$-ray bending magnet (XRBM), and the NSLS X1 undulator (X1). Note that the total output power loads from these three sources are much more similar than are their peak power densities. This observation is graphically presented in Fig. 1, which shows the incident power density contours on the front face of the mirror described above. The peak power density for $\mathrm{X} 1\left(1040 \mathrm{~W} / \mathrm{mrad}^{2}\right.$ at $\left.\mathrm{E}=2.5 \mathrm{GeV}, \mathrm{I}=200 \mathrm{~mA}, \mathrm{~K}=2.5\right)$ is 32 times that for $\mathrm{U} 13$ $\left(32.5 \mathrm{~W} / \mathrm{mrad}^{2}\right.$ at $\mathrm{E}=0.75 \mathrm{GeV}, \mathrm{I}=500 \mathrm{~mA}, \mathrm{~K}=8$ ), whereas the total power for these two devices is similar (XI: $262 \mathrm{~W}, \mathrm{U13}: 287 \mathrm{~W}$ ). The peak power density for an NSLS XRBM source $\left(51.7 \mathrm{~W} / \mathrm{mrad}^{2}\right.$ at $\left.E=2.5 \mathrm{GeV}, I=200 \mathrm{~mA}\right)$ is $5 \%$ of that for $\mathrm{X} 1$, but within the horizontal angle collected by the mirror described above (6.5 mrad), the 
power output from XRBM (105 W) is $40 \%$ of that from X1. Notice that the very small vertical opening angle $\left(\sigma_{r}\right)$ of these synchrotron radiation devices causes the foreshortening of the incident power distribution to be negligible, even at $2^{\circ}$ grazing angle of incidence.

\section{Results of thermal and stress analysis}

In this section we present finite element analyses of the $\mathrm{SiC}$ mirror described above under power loading. We choose the mirror described in sec. 1 to be the second optical element $\left(\mathrm{M}_{1}\right)$ in the beamline, the first being a horizontal collecting and focusing mirror $\left(\mathrm{M}_{0}\right)$ at $3^{\circ}$ grazing angle of incidence, as in the SGM currently being constructed for the U13 wiggler/undulator spectroscopy branch line at the NSLS. The $\mathbf{M}_{0}$ mirror will thermally deform mainly in the sagittal direction, with relatively little effect on the focusing properties of the $M_{1}$ mirror. This is fortunate because the quality of the tangential focus of the $M_{1}$ mirror determines the flux throughput of the entrance slit, which is a major factor in the efficiency of the entire beamline. The use of separate horizontal and verical focusing optical elements derives originally from Kirkpatrick and Baez [7] and has been used in beamlines based on the Grasshopper [8] and Extended Range Grasshopper (ERG) $[9,10]$ soft $x$-ray grazing incidence monochromators, among others. The soft $x$-ray reflectivity of a gold coating at $3^{\circ}$ grazing angle of incidence is $=50 \%$ or greater below $1000 \mathrm{eV}$ (independent of polarization, to close approximation), so we assume that $50 \%$ of the source ourput power is absorbed by $M_{0}$. This value is not a bad approximation for the case of the U13 wiggler, which has a maximum critical energy of $320 \mathrm{eV}$ : only $5.5 \%$ of the output power from this device is contributed by photons with energy greater than $1000 \mathrm{eV}$. However, this assumption underestimates the $M_{0}$ heat load for an X1 source: treating this source as a wiggler ( $K$ $=2.5$ ), the maximum critical energy is $1370 \mathrm{eV}$ and $=1 / 2$ of the $X 1$ output power is contributed by photons with energy greater than $1000 \mathrm{eV}$. Thus, $\mathbf{M}_{0}$ actually reflects 
only $=25 \%$ of the $\mathrm{X} 1$ output power rather than the $50 \%$ value assumed in these caiculations; conversely, these calculations correspond to the $\mathrm{X} 1$ source when the NSLS Xray ring is operating at $400 \mathrm{~mA}$ stored current rather than the $200 \mathrm{~mA}$ value shown in Table I. Finally, this assumption largely underestimates the power absorbed by $\mathrm{M}_{0}$ for an XRBM source, which has a critical energy of $5.1 \mathrm{keV}$, and distorts its spatial distribution. Clearly, specific power loading cases for XRBM sources should be dealt with separately, but the present calculation (which assumes $50 \% \mathrm{M}_{0}$ reflectivity for this source as well) demonstrates the heat loading effect of a source which has roughly (within a factor of 2) the same total power incident upon $\mathrm{M}_{1}$ as for $\mathrm{U} 13$ and $\mathrm{X} 1$ but is spatially uniform in the orbital (horizontal) plane. The average reflectivity for a $2^{\circ}$ grazing gold mirror is $=60 \%$, so we assume that $40 \%$ of its input power is absorbed. Therefore, the $\mathrm{M}_{1}$ mirror considered here absorbs approximately $20 \%$ of the source output power (Fig. 1 shows incident power contours). We assume that the spatial distribution of the absorbed power is the same as that shown in Fig. 1, a good assumption for the $U 13$ and $X 1$ sources.

The heat flow from the front face of the $\mathrm{M}_{1}$ mirror to the cooling channels in the copper side rails is essentially three-dimensional spatially. Therefore, a 3-D finite element model using ANSYS ${ }^{\dagger}$ was prepared for the heat transfer analyses. The same model with some modifications was subsequently used to determine the deformations and stresses in the mirror. Because of the twofold symmetry about the center of the front face, only a quarter of the mirror was modeled. The finite element model, which consists of 840 elements in the mirror and 378 elements in each copper rail, is shown in Fig. 2. Square channels with the same effective cooling surface area as the circular channels in the actual design were used in order to reduce the complexity of the model. A water flow of $3 \mathrm{~m} / \mathrm{s}$ at $25^{\circ} \mathrm{C}$ was assumed in the channels, which is equivalent to a film coefficient of $0.013 \mathrm{~W} \mathrm{~mm}^{-2}{ }^{\circ} \mathrm{C}^{-1}$. The thermal conductivity

\footnotetext{
† ANSYS -- A General Pupose Finite Element Program. Rev. 4.2. Swonson Analysis Systems, Ine., Hourron. Pennsylvanis.
} 
values for $\mathrm{SiC}$ and $\mathrm{Cu}$ were taken as 0.126 and $0.390 \mathrm{~W} \mathrm{~mm}^{-1}{ }^{\circ} \mathrm{C}^{-1}$, respectively. The analysis results show that the maximum temperature difference $(\Delta T)_{\max }$ produced in this mirror is $0.6^{\circ} \mathrm{C}$ for $\mathrm{U} 13,0.9^{\circ} \mathrm{C}$ for $\mathrm{XRBM}$, and $5.8^{\circ} \mathrm{C}$ for $\mathrm{X} 1$ (see Table II). Although elevated temperatures alter the rates of surface chemical reactions such as the undesirable cracking of residual hydrocarbons, at these modest temperatures $\left(5.8^{\circ} \mathrm{C}\right.$ maximum temperature difference) the most important effect by far is the deformation caused by the intemal stresses resulting from the thermal gradients in the mirror. In particular, it is the deformations normal to the front surface of the mirror that most affect its optical performance, especially at the grazing angles of incidence used in most VUV/soft $x$-ray beamlines. At grazing incidence, tangential slope errors have a very much larger effect on focusing in the tangential plane than do sagittal slope errors. Element temperatures obtained from the heat transfer analyses were used to determine thermal stresses and deformations in the SiC mirror. For these calculations, the copper rail was ignored and the mirror was assumed to be fixed at the edges of the back face. The following material properties were used for SiC: thermal coefficient of expansion $\alpha=4.02 \times 10^{-6} \mathrm{~mm}$ per $\mathrm{mm}^{\circ} \mathrm{C}$, elastic modulus $E=59.0 \times 10^{3} \mathrm{ksi}(1 \mathrm{ksi}=$ $6.895 \times 10^{6} \mathrm{~Pa}$ ), and Poisson's ratio $v=0.142$.

Normal displacement contour plots are shown in Fig. 3 for each of the three synchrotron radiation sources shown in Fig. 1. Note the large differences in contour shape and density for these sources, corresponding to the large differences in absorbed power density. The average displacement contour densities (i.e. the average optical figure errors) are not proportional to the maximum temperature differences listed above: the average slope error in the tangential (focusing) direction (along the length of the mirror) is $0.1 \operatorname{arcsec}$ for XRBM, $0.1 \operatorname{arcsec}$ for U13, and $0.3 \operatorname{arcsec}$ for X1. The maximum slope error is generally larger than the average slope error, especially for the $\mathrm{X} 1$ undulator owing to its highly collimated output: the maximum tangential slope errors are 0.1 arcsec for U13 [Fig. 3(a)], 0.25 arcsec for XRBM [Fig. 3(b)], and 0.9 
arcsec for X1 [Fig. 3(c)]. Note that the maximum tangential slope errors are also not proportional to $\Delta \mathrm{T}_{\max }$. The average and maximum tangential slope error values are listed in Table II, along with the maximum temperature differences. The internal stresses in the mirror (not shown in Table II) are very small because of the small temperature differences; e.g. the maximum stress for U13 power loading is only 172 psi.

As can be seen from Table $\mathrm{I}$, these is no simple relationship between the total input power $P$ and either the average or maximum resulting tangential slope error. However, for a given mirror material (e.g. SiC), there exists a direct, but not linear, relationship between the maximum input power density $\left(\frac{d P}{d \theta d \psi}\right)_{\max }$ and the average and maximum tangential slope errors, as well as with the maximum temperature difference $(\Delta \mathrm{T})_{\max }$

Figure 4 is the normal displacement contour plot for an aluminum mirror under $\mathrm{XI}$ power loading, with all other parameters the same as for the SiC mirror. Whereas the maximum temperature difference for the aluminum mirror is $3.8^{\circ} \mathrm{C}$ (less than for $\mathrm{SiC}$ under $\mathrm{X} 1$ loading), the average tangential slope error is 2.4 aresec and the maximum tangential slope error is 6.1 arcsec (almost 7 times that for $\mathrm{SiC}$ under $\mathrm{X} 1$ loading). Thus, the figure error thermally induced in mirrors of different materials for a given absorbed power distribution is roughly inversely proportional to the deformation figure of merit $\mathrm{K} / \alpha$ described in sec. 2.

\section{Effect on optical performance}

The monochromators of the next generation of VUV/soft $x$-ray synchrotron radiation beamlines are designed to provide up to an order of magnitude higher resolving power $(E / \Delta E)$ than monochromators on existing beamlines without sacrificing transmission (flux throughput). The ability of all of these new beamlines to achieve this goal is limited in varying degrees by the figure errors present in the surfaces of their optical elements. Thermally-induced figure error should be kept lower than that achievable by 
the best optical companies, which is presently near 0.5 arc sec for large radius spherical mirrors and grating blanks. Clearly, an aluminum mirtor does not meet this criterion for X1 power loading, but a SiC mirror does meet it for all three synchrotron radiation sources considered in this paper (XRBM, U13, and X1). In this section we present ray tracing results using a ray tracing program developed by Cerrina ${ }^{\dagger}$ specifically for synchrotron radiation beamlines in order to make quantitative statements concerning the effects of mirtor figure error on the transmission of the U13 SGM and the energy resolution of the UMO monochromator. Due to the presence of an entrance slit in the SGM design, figure error in the vertical focusing mirror $M_{1}$ produces a loss in transmission at the entrance slit but does not affect the resolving power E/AE of the monochromator. To this extent, the results presented below for the SGM also apply to the Grasshopper, ERG, and toroidal grating (TGM) [11-13] monochromators (albeit with different magnifications depending on the geometry and figure of the collecting and focusing optics upstream of the entrance slit). In contrast, thermallyinduced optical figure error in beamlines based on the proposed UMO monochromator and the SX700 monochromator of Petersen $[14,15]$, which do not urilize an entrance slit, results in both a loss in transmission and a reduction in resolving power.

Again, an entrance slit provides stability in the selected photon energy hv against movement of the photon beam incident upon it at the cost of a possible reduction in transmitted flux. Therefore, the effect of thermal deformation of the optical elements upstream of the entrance slit in the SGM is a reduction in flux, with the selected photon energy $h v$ and energy resolution $\Delta E$ remaining essentially unchanged. The heat load issues for the entrance slit and the grating, which are not considered in detail here, are significant: a change in entrance slit width directly affects the energy resolution $\Delta \mathrm{E}$, as do grating figure errors. Obviously, if the front-end mirror heat load problems can be solved adequately, the slit and grating heat load problems will then

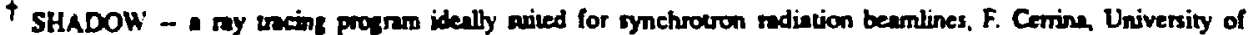
Wisconsin, Madison, Wisconsin
} 
dominate. Histograms of the image in the focal direction on an entrance slit $5 \mathrm{~m}$ downstream of the $M_{1}$ mirror described in sec. 2 are presented in Fig. 5 for average mirror tangential slope errors of $0,1,2,5$, and 10 arcsec. The radius $R$ of the mirror is chosen to be $191 \mathrm{~m}$ so as to produce the best point-to-point focus onto the entrance slit. The sourc: for these calculations is Gaussian in both space $\left(\sigma_{x}=1.2 \mathrm{~mm}, \sigma_{y}=\right.$ $0.1 \mathrm{~mm})$ and angle $\left(\sigma_{x^{\prime}}=3.25 \mathrm{mrad}, \sigma_{y^{\prime}}=0.9 \mathrm{mrad}\right)$, such that the mirror considered here is filled. Of course, the actual emittance from the various types of synchrotron radiation devices varies greatly; Fig. 5 demonstrates the dependence on figure error alone, with all other parameters remaining fixed. Gaussian fits in the tangenial and sagittal directions are used in the ray tracing calculations to approximate the surface normal displacements calculated in sec. 3 for U13 (Fig. 5, upper) and X1 (Fig. 5 , lower) power loading. Figure 5 demonstrates that, for a typical slit width of $100 \mu \mathrm{m}$, the transmission of the entrance slit under U13 power loading is reduced by $2 \%$ for 1 arcsec, by $10 \%$ for 2 arcsec, by $40 \%$ for 5 arsec, and by $67 \%$ for 10 arcsec average tangential slope error. The corresponding flux losses for $\mathrm{X} 1$ power loading are $10 \%$ for 1 arssec, $30 \%$ for 2 arcsec, $55 \%$ for 5 arcsec, and $67 \%$ for 10 arcsec average tangential slope errors. Note that the histograms vary in intensity and peak position as a function of both average tangential slope srror ( 0 to 10 aresec) and the shape of the displacement profile (U13 vs. X1). The flux is essentially unchanged for tangential slope errors less than 0.5 arcsec, so we conclude that the restrained and cooled $\mathrm{SiC} \mathrm{M}_{1}$ mirror considered in this paper will produce very little loss in flux at the entrance slit of the SGM for U13, XRBM, and X1 sources. Under X1 power loading, the normal displacements of an aluminum $M_{1}$ miror (Fig. 4) are almost an order of magnitude greater than a $\mathrm{SiC} \mathrm{M}_{1}$ mirror [Fig. 3(c)], but the shapes of the normal displacement profiles are very similar. For the aluminum $\mathrm{M}_{1}$ mirror, the resulting reduction in flux at the entrance slit of the SGM is $\approx 30 \%$. These flux reduction figures depend strongly on the source vertical opening angle $\sigma_{y^{\prime}}$, so adjustment for specific source characteris- 
tics is required.

Virtual source monochromator designs, such as that of the UMO and SX700, provide the possibility of high energy resolution and high diffraction efficiency over a large range of wavelengths. The absence of an entrance slit, however, aliows figure errors on the first mirror to distort the distribution of angles of incidence on the grating and thereby distort the photon energy bandwidth selected by the exit slit. This distortion of the UMO monochromator bandpass is shown in Fig. 6. The UMO parameters chosen for these calculations are $C=2.25, \alpha=88^{\circ}$, grating groove spacing 1200 lines/mm, angle of incidence on the first mirror $\left(M_{1}\right)=86.75^{\circ}$, angle of incidence on refocising ellipsoid $=88.003^{\circ}$, and exit slit width $=50 \mu \mathrm{m}$ (producing resolving power $E / \Delta E=400$ ), and $M_{1}$ is $10 \mathrm{~m}$ from the synchrotron source as for the SGM discussed above. The source for these calculations is Gaussian in both space and angle with the same widths as used for the SGM calculations above except that $\sigma_{x^{\prime}}=1 \mathrm{mrad}$ is used in order to reduce coma effects from the refocusing ellipsoid. Figure 6 shows photon wavelength histograms centered about the selected wavelength $\lambda=20.655 \AA \mathrm{hv}=$ $600 \mathrm{eV}$ ) for average $M_{1}$ tangential slope errors of $0,1,2,5$, and 10 arcsec for U13 (Fig. 6, upper) and X1 (Fig. 6, lower) power load distributions. Figure 6 shows that the $M_{1}$ figure error produced by these power loads causes a gradual broadening of the wavelength bandpass and a reduction in transmitted flux. For 1 and 2 arcsec average $M_{1}$ tangential slope errors, the flux and bandpass of the UMO monochromator is essentially unchanged from that of a perfect $M_{1}$ mirror. For U13 power loading, the bandwidth is increased by $=25 \%$ for 5 arcsec and by almost a factor of 2 for 10 arcsec average tangential slope error; and the flux within the bandwidth selected by a perfect $M_{1}$ mirror $(0.05 \AA)$ drops by $10 \%$ for 2 arcsec, $20 \%$ for 5 arcsec, and $50 \%$ for 10 arcsec average tangential slope error. For $\mathrm{X} 1$ power loading, the bandwidth increases by $15 \%$ for 2 arcsec, by a factor of 2 for 5 arcsec, and by a factor of 3 for 10 arcsec average tangential slope error; the flux within the zero-figure-error bandwidth drops by 
$20 \%$ for $2,40 \%$ for 5 , and $60 \%$ for 10 arcsec average tangential slope errors. The restrained and cooled $\mathrm{SiC} \mathrm{M}_{1}$ mirror considered in this paper deforms less than 0.5 arcsec (average) under U13, XRBM, and X1 power loading, and this would not (within $=10 \%$ ) degrade the resolving power or flux through the UMO monochromator with $50 \mu \mathrm{m}$ exit slit. For an alumirum $\mathrm{M}_{1}$ mirror under $\mathrm{X} 1$ power loading, however, the resolving power of the UMO would decrease by approximately $20 \%$.

If no cooling is used, the temperature of the mirror rises considerably (250 to $300^{\circ} \mathrm{C}$ for U13), but the maximum temperature differences remain close to the values listed in sec. 3 and Table II (although temperature-induced vacuum problems may result). If the mirror is not restrained, however, the deformations are much larger than those shown in Fig. 3, by a factor of $=20$ (tangential slope error $=2.3$ arcsec for a SiC $M_{1}$ mirror under $U 13$ power loading).

\section{Conclusions}

We have presented a set of thermal stress analyses of a $\mathrm{SiC}$ grazing incidence soft $\mathrm{x}$-ray mirror subject to the power loads of three synchrotron radiation sources at the NSLS: the U13 wiggler/undulator, an $\mathrm{X}$-ray bending magnet, and the X1 undulator. We chose a specific geometry in order to allow comparisons between different sources. The results of the 3-D finite-element analyses allow us to conclude that whereas the calculated surface normal deformations are not directly related to the total input power - P, they are directly (although not linearly) related to the maximum input power density $\left(\frac{d P}{d \theta d \phi}\right)$ and to the maximum temperature difference $(\Delta T)_{\max }$. In addition, the deformations for mirrors of different materials with the same input power distributions vary approximately inversely as the deformation figure of merit $(\mathrm{K} / \alpha)$. The deformations for back-edge-restrained, side-cooled SiC mirrors under these three power loads all correspond to tangential slope errors less than 1 arcsec. We have shown that this does not (within $\pm 5 \%$ ) limit the flux through a $\leq 100 \mu \mathrm{m}$ entrance slit located $5 \mathrm{~m}$ down- 
stream of this mirror. Therefore the SGM, as well as the Grasshopper, ERG, and TGM monochromators, and others that utilize an entrance slit, would not experience a loss in flux (or resolution) under these power loading conditions. An aluminum mirror under $\mathrm{X} 1$ loading, however, would reduce the flux through an entrance slit by $1 / 3$ due to its poorer deformation figure of merit. We have also shown that these deformations of the restrained and cooled $\mathrm{SiC} \mathrm{M}_{1}$ mirror would not (within $\pm 5 \%$ ) reduce the resolving power or transmitted flux of the UMO monochromator operating with 50 pm exit slit $(E / \Delta E=400$ at $\lambda=20.655 \AA)$ but that an aluminum $M_{1}$ mirror under $X 1$ loading would reduce the UMO resolving power by $=20 \%$.

The deformations under power loading from undulators or wigglers on a higher energy machine (e.g. $6 \mathrm{GeV}$ ) would be much larger than those resulting from the sources considered here. More sophisticated cooling schemes [16] will be required: for these sources. To the extent that the power loading on the first and second mirrors can be solved, the dominant heat load problems in soft $\mathrm{x}$-ray beamlines will be on the entrance slit and on the gratings. A total power load of $300 \mathrm{~W}$ focused onto a $100 \mu \mathrm{m}$ $\times 1 \mathrm{~cm}$ slit is a power density of $30 \mathrm{~kW} / \mathrm{cm}^{2}$, which can cause much distortion if directed onto either slit jaw. If the entrance slit passes most of the power incident upon it (as desired), then the power load on the gratings will be a major problem in both current and future generation synchrotron radiation sources since it directly affects both the resolution and flux throughput in all currently designed soft $\mathbf{x}$-ray beamlines.

\section{Acknowledgment}

This work was supported by the Division of Material Sciences, U. S. Department of Energy, under Contract DE-ACO2-76CH00016. 


\section{References}

1. R. Z. Bachrach, R. D. Bringans, N. Hower, I. Lindau, B. B. Pate, P. Pianetta, L. E. Swartz, and R. Tatchyn, "Design Process and Modeling Studies of SSRL Beam Line Wunder", Nucl. Instrum. Meth. 222 , 70-79 (1984); W. R. Hunter and R. T. Williams, "Grain Boundary Diffusion of Liquid Metal Coolants in Optical Materials for use with High Power Synchroton Radiation", Nucl. Instrum. Meth. 222 , 359-363 (1984); see also section II(g) of Nucl. Instrum. Meth. A246 (1986).

2. C. T. Chen, "Concept and Design Procedure for Cylindrical Element Monochromators for Synchrotron Radiation", Nucl. Instrum. Meth. A256 , 595-604 (1987).

3. F. C. Brown and S. L. Hulbert, "A High Resolution Monochromator for Undulator Radiation", Nucl. Instrum. Meth. 222 , $42-45$ (1984).

4. F. R. Holdener, E. J. Berglin, B. A. Fuchs, H. H. Humpal, V. K. Karpenko, R. W. Martin, and K. G. Tirsell, "Thermal Loading Considerations for Synchrotron Radiation Mirrors", in Optics and Optoelectronic Systems, J. Osantowski and L. Van Speybroeck, eds.,

Proc. SPIE 640,116 (1986).

5. K.-J. Kim, "Angular Distribution of Undulator Power for an Arbitrary Deflection Parameter K", Nucl. Instrum. Meth. A246 , 67-70 (1986).

6. G. K. Shenoy and P. J. Viccaro, "An Overview of the Characteristics of the 6-GeV : Synchrotron Radiation: A Preliminary Guide for Users", Argonne National Labora- . tory report ANL-85-69.

7. P. Kirkpatrick and A. V. Baez, "Formation of Optical Images by X-Rays", J. Opt. Soc. of Amer. $38,766-774$ (1948).

8. F. C. Brown, R. F. Bachrach, and N. Lien, "The SSRL Ultrahigh Vacuum Grazing Incidence Monochromator: Design Considerations and Operating Experience", : Nucl. Instrum. Meth. 152,73 (1978). 
9. S. L. Hulbert, J. P. Stott, and F. C. Brown, "An Extended Range Soft X-ray Beam Line for the $1 \mathrm{GeV}$ Storage Ring Aladdin", Nucl. Instrum. Meth. 208 , 43-47 (1983).

10. F. C. Brown, J. P. Stott, and S. L. Hulbert, "First Operation of an Extended Range Grasshopper Monochromator on the Aladdin Storage Ring", Nucl. Instrum. Meth. A246, 278-281 (1986).

11. D. Lepere, Nouv. Rev. Oyt. 6,173 (1975); see review paper by R. L. Johnson in Handbook on Synchrotron Radiation, Vol. 1, ed. E. E. Koch, North-Holland, 1983.

12. B. P. Tonner, "Performance of a Dual Toroidal Grating Monochromator", Nucl. Instrum. Meth. $172,133-135(1980)$.

13. C. T. Chen, E. W. Plummer, and M. R. Howells, "The Study and Design of a High Transmission, High Resolution Toroidal Grating Monochromator for Soft Xray Radiation', Nucl. Instrum. Meth. 222 , 103-106 (1984).

14. H. Petersen, "The Plane Grating and Elliptical Mirror: A New Optical Configuration for Monochromators", Opt. Comm. 40 , 402-406 (1982).

15. H. Petersen, "The High Energy Plane Grating Monochromators at BESSY", Nucl. Instrum. Meth. $A 246,260-263$ (1986).

16. R. DiGennaro, B. Gee, J. Guigli, H. Hogrefe, M. R. Howells, and H. Rarback, "A Water-cooled Mirtor System for Synchrotron Radiation', Nucl. Instrum. Meth., to be published. 


\section{Figure Captions}

1. Incident power density contow plots for $a$ vertically-deflecting mirror surface at $2^{\circ}$ grazing angle of incidence corresponding to three synchrotron radiation sources at the NSLS: (a) the U13 hybrid wiggler/undulator, (b) an $\mathrm{x}$-ray bending magnet (XRBM), and (c) the X1 undulator. Although the radiation intercepts the mirror from the left, the vertical opening angle $\sigma_{y^{\prime}}$ of these sources is so small that foreshortening is negligible: all three contour patterns are essentially symmetric about the center of the minor (the axis of the radiation pattern). In (a) and (b), the contour spacing is $0.2 \mathrm{~W} / \mathrm{cm}^{2}$ and contours are drawn bold for values greater than $0.5 \mathrm{~W} / \mathrm{cm}^{2}$. In (c), the contour spacing is $2.0 \mathrm{~W} / \mathrm{cm}^{2}$ and contours are drawn bold for values greater than $5.0 \mathrm{~W} / \mathrm{cm}^{2}$. The value of the maximum contour is 1.0 $\mathrm{W} / \mathrm{cm}^{2}$ in (a), $1.8 \mathrm{~W} / \mathrm{cni}^{2}$ in (b), and $36.0 \mathrm{~W} / \mathrm{cm}^{2}$ in (c).

2. 3-D finite element model used for thermal analyses of the side-cooled backrestrained $\mathrm{SiC}$ mirror. The synchrotron radiation is incident from the left at $2^{\circ}$ grazing angle of incidence on the $x-y$ (front) face. The three thinner $x-z$ planes of tiles on the outside face of the mirror represent the copper cooling rails. The two rectangular water channels are indicated.

3. Surface normal displacement contour plots resulting from the absorption of $20 \%$ of the corresponding incident power load distributions shown in Fig. 1 by the sidecooled back-edge SiC mirror. The contour spacing is $250 \AA$ in all three plots, and contours are drawn bold for displacements of $500 \AA$ and greater. The maximum displacement contour values are $1000 \AA$ for U13 (a), $1000 \AA$ for XRBM (b), and $3750 \AA$ for $\mathrm{X} 1$ (c). The corresponding average and maximum tangential slope errors are listed in Table $\Pi$. Only one quadrant of the mirror is shown; the other three can be generated by reflections about the $\mathrm{x}$ and $\mathrm{y}$ axes.

4. Surface normal displacement contour plot resulting from the absorption of $20 \%$ of the incident power load distribution from the Xl undulator shown in Fig. I(c) by 
the side-cooled back-edge-restrained Al mirror. The contour spacing is $250 \AA$, contours are drawn bold for displacements of $500 \AA$ and greater, and the maximum displacement contour value is $29000 \AA$. The corresponding average and maximum tangential slope errors are listed in Table II. Only one quadrant of the mirror is shown; the other three can be generated by reflections about the $x$ and $y$ axes.

5. Histograms in the tangential (vertical) direction on the focal plane of the $2^{\circ}$ grazing angle of incidence spherical mirror. Source distance is $10 \mathrm{~m}$, image distance is 5 $\mathrm{m}$, and mirror radius is $191 \mathrm{~m}$. In decreasing order of intensity, the average tangential slope errors shown are $0,1,2,5$, and 10 arcsec for both the upper and lower figures. The displacement spatial distributions used in the upper and lower figures are Gaussian fits in the tangential and sagittal directions to the U13 and X1 displacement distributions shown in Fig. 3(a) and Fig. 3(c), respectively.

6. Photon wavelength histograms centered about the selected wavelength $\lambda=20.655 \AA$ (hv $=600 \mathrm{eV}$ ) for the UMO monochromator. In decreasing order of intensity, the average tangential slope errors shown are $0,1,2,5$, and 10 arcsec for both the upper and lower figures. The displacement sparial distribution used in the upper and lower figures are Gaussian fits in the tangential and sagittal directions to the $\mathrm{U} 13$ and $\mathrm{X} 1$ dispiacement distributions shown in Fig. 3(a) and Fig. 3(c), respectively. 
Table 1. Device parameters, total power, and maximum power density for various bending magnel and inserion device synchrotron radiation sources.

Bending Magnet

\begin{tabular}{l|c|c|c|c|c|c|c} 
Source & $E_{R}(\mathrm{GeV})$ & $B(T)$ & $I(m A)$ & $\rho(\mathrm{cm})$ & $E_{c}(\mathrm{keV})$ & $\begin{array}{c}P \\
(W / \mathrm{mrad})\end{array}$ & $\begin{array}{l}{\left[\mathrm{d}^{2} \mathrm{P} /(\mathrm{d} \theta \mathrm{d} \psi)\right]_{\max }} \\
\left(\mathrm{W} / \mathrm{mmad}^{2}\right)\end{array}$ \\
\hline NSLS UV & 0.75 & 1.28 & 500 & 1.91 & 0.48 & 1.11 & 1.10 \\
NSLS XR* & 2.5 & 1.22 & 200 & 6.88 & 5.07 & 16.2 & 51.7 \\
$6 \mathrm{GeV}$ & 6.0 & 0.67 & 100 & 30.0 & 16.04 & 61.2 & 471.
\end{tabular}

Wiggler or Undulator

\begin{tabular}{l|l|l|l|l|l|l|r|r} 
Source & $E_{R}(\mathrm{GeV})$ & $B_{0}(\mathrm{~T})$ & $\mathrm{I}(\mathrm{mA})$ & $\lambda_{\mathrm{u}}(\mathrm{cm})$ & $\mathrm{K}$ & $\mathrm{N}$ & $\mathrm{P}(\mathrm{W})$ & $\begin{array}{l}{\left[\mathrm{d}^{2} \mathrm{P} /(\mathrm{d} \theta \mathrm{g} \psi)\right]_{\max }} \\
\left(\mathrm{W} / \mathrm{mrad}^{2}\right)\end{array}$ \\
& & & & & & & & \\
NSLS U5 & 0.75 & 0.16 & 500 & 6.5 & 1 & 38 & 11.9 & 10.4 \\
NSLS U13* & 0.75 & 0.86 & 500 & 10.0 & 8 & 22 & 287. & 32.5 \\
NSLS X1* & 2.5 & 0.33 & 200 & 8.0 & 2.5 & 37 & 262. & 1040. \\
NSLS X17 & 2.5 & 6.0 & 200 & 17.4 & 97.5 & 6 & 29700. & 3050. \\
6 GeV wiggler & 6.0 & 1.5 & 100 & 10.0 & 14 & 30 & 15400. & 63300. \\
6 GeV undulator & 6.0 & 0.16 & 100 & 19.8 & 3 & 20 & 230. & 4500.
\end{tabular}

- Synchrotron radiation sources considered in this study.

Table II. Summary of power loads, material properties, maximum temperature differences, and resulting average and maximum slope errors considered in this paper.

\begin{tabular}{l|c|c|c|c|c|c|c} 
Source & $\begin{array}{c}P \\
(W)\end{array}$ & $\begin{array}{l}{\left[\mathrm{d}^{2} \mathrm{P} /(\mathrm{d} \theta \mathrm{d} \psi)\right]} \\
\left(\mathrm{W} / \mathrm{mrad}^{2}\right)\end{array}$ & Material & $\begin{array}{c}\mathrm{K} / \mathrm{a} \\
\left(10^{4} \mathrm{~W} / \mathrm{cm}\right)\end{array}$ & $\begin{array}{c}(\Delta T)_{\max } \\
\left({ }^{\circ} \mathrm{C}\right)\end{array}$ & $\begin{array}{c}\text { Ave. slope error } \\
(\operatorname{arc~sec})\end{array}$ & $\begin{array}{c}\text { Max. slope error } \\
\text { (arc sec) }\end{array}$ \\
$\mathrm{U13}$ & 287 & 32.5 & $\mathrm{SiC}$ & 31.3 & 0.6 & 0.1 & 0.1 \\
$\mathrm{XRBM}$ & 105 & 51.7 & $\mathrm{SiC}$ & 31.3 & 0.9 & 0.1 & 0.25 \\
$\mathrm{X} 1$ & 262 & 1040. & $\mathrm{SiC}$ & 31.3 & 5.8 & 0.3 & 0.9 \\
$\mathrm{X} 1$ & 262 & 1040. & $\mathrm{Al}$ & 7.0 & 3.8 & 2.4 & 6.1
\end{tabular}



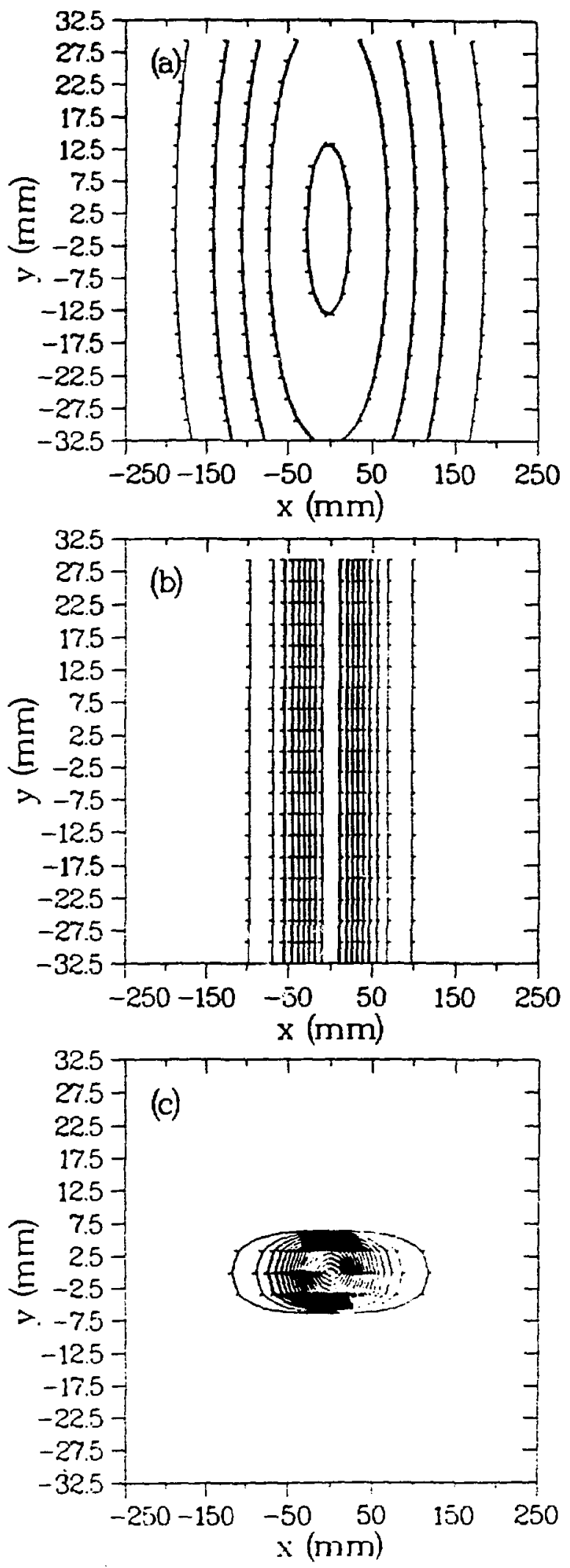


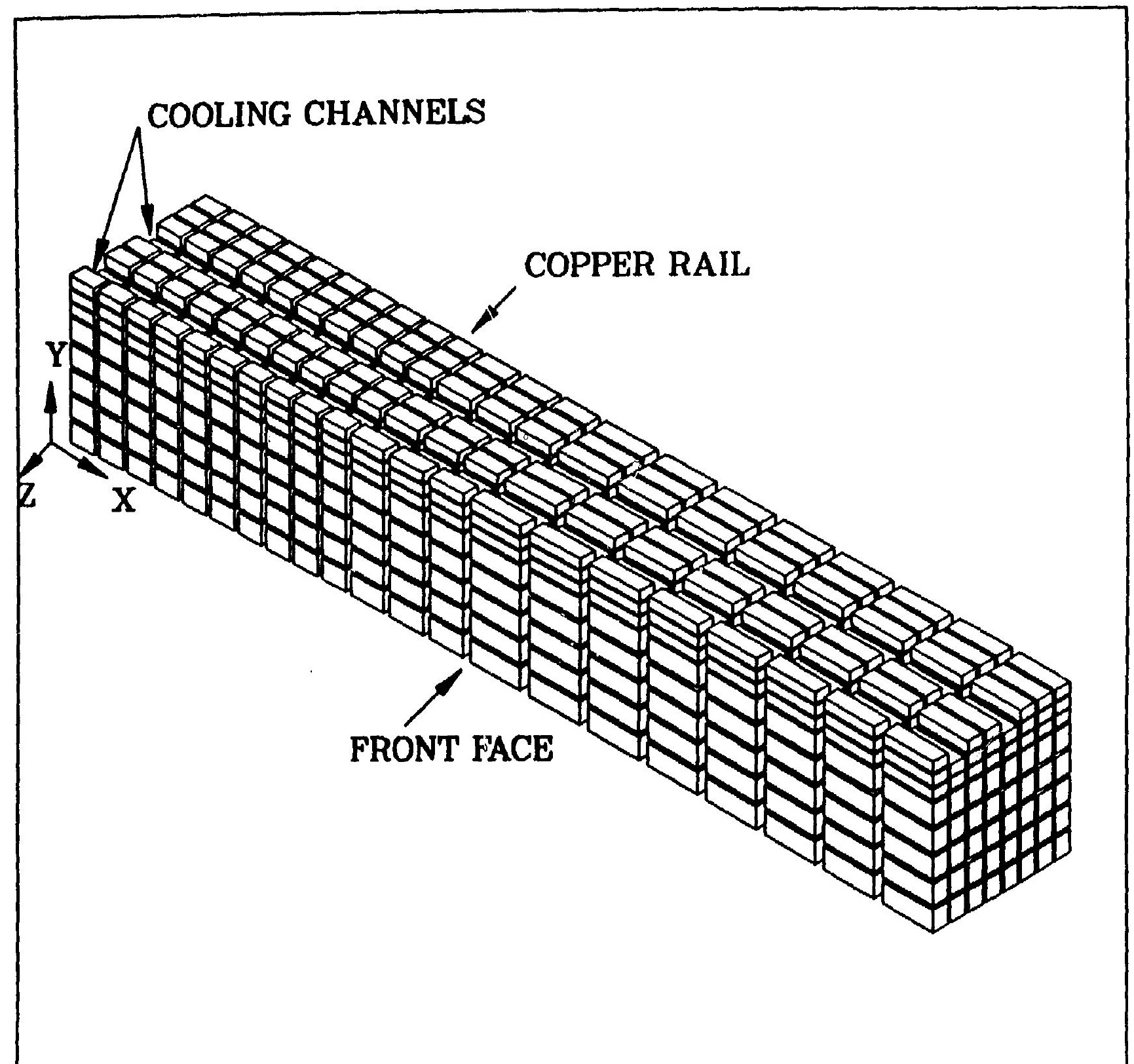



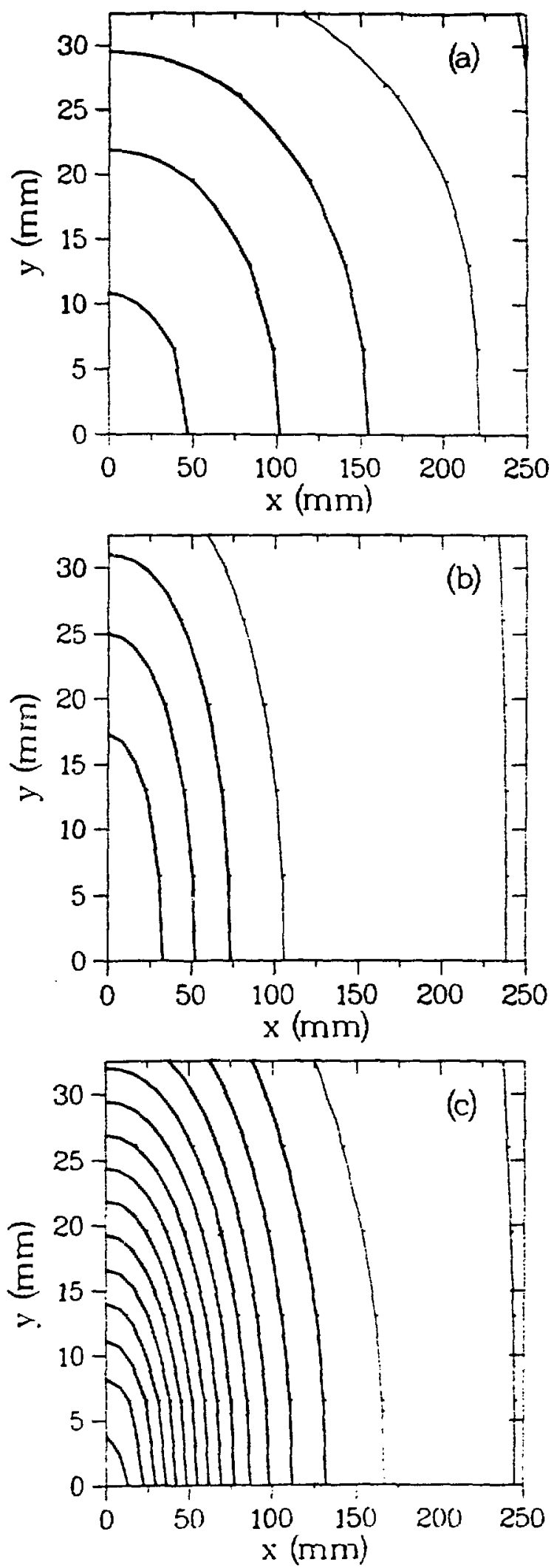


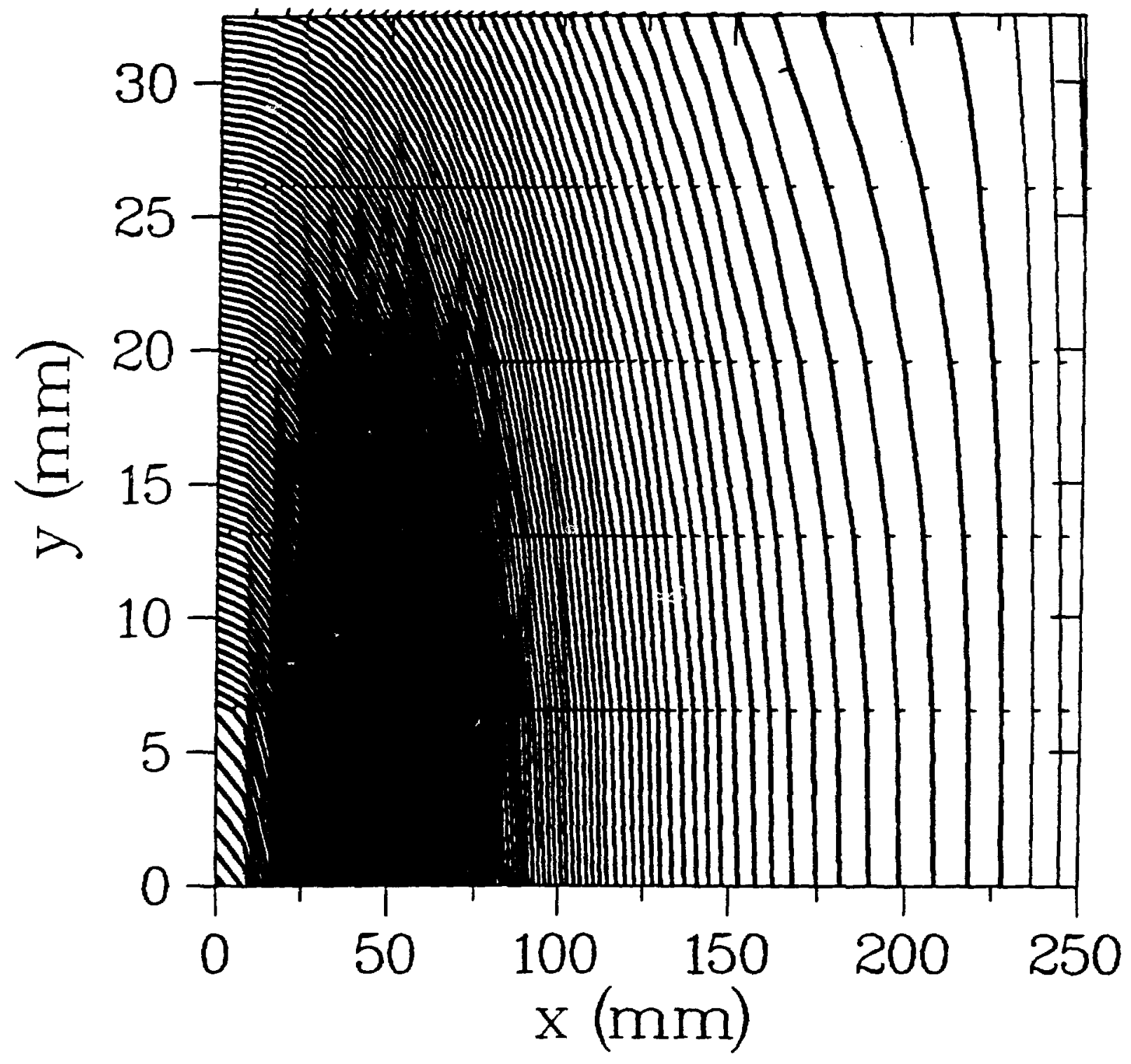



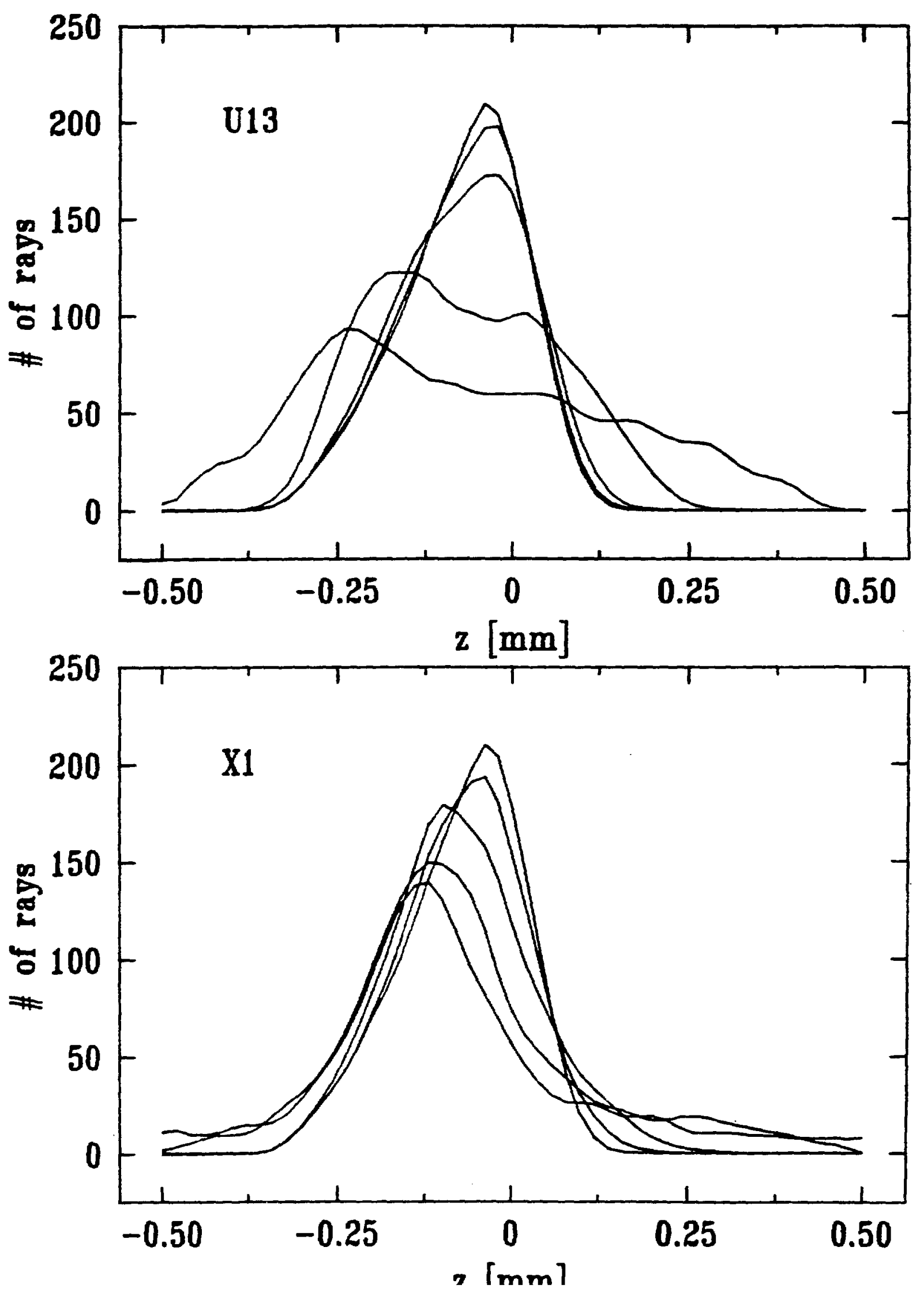

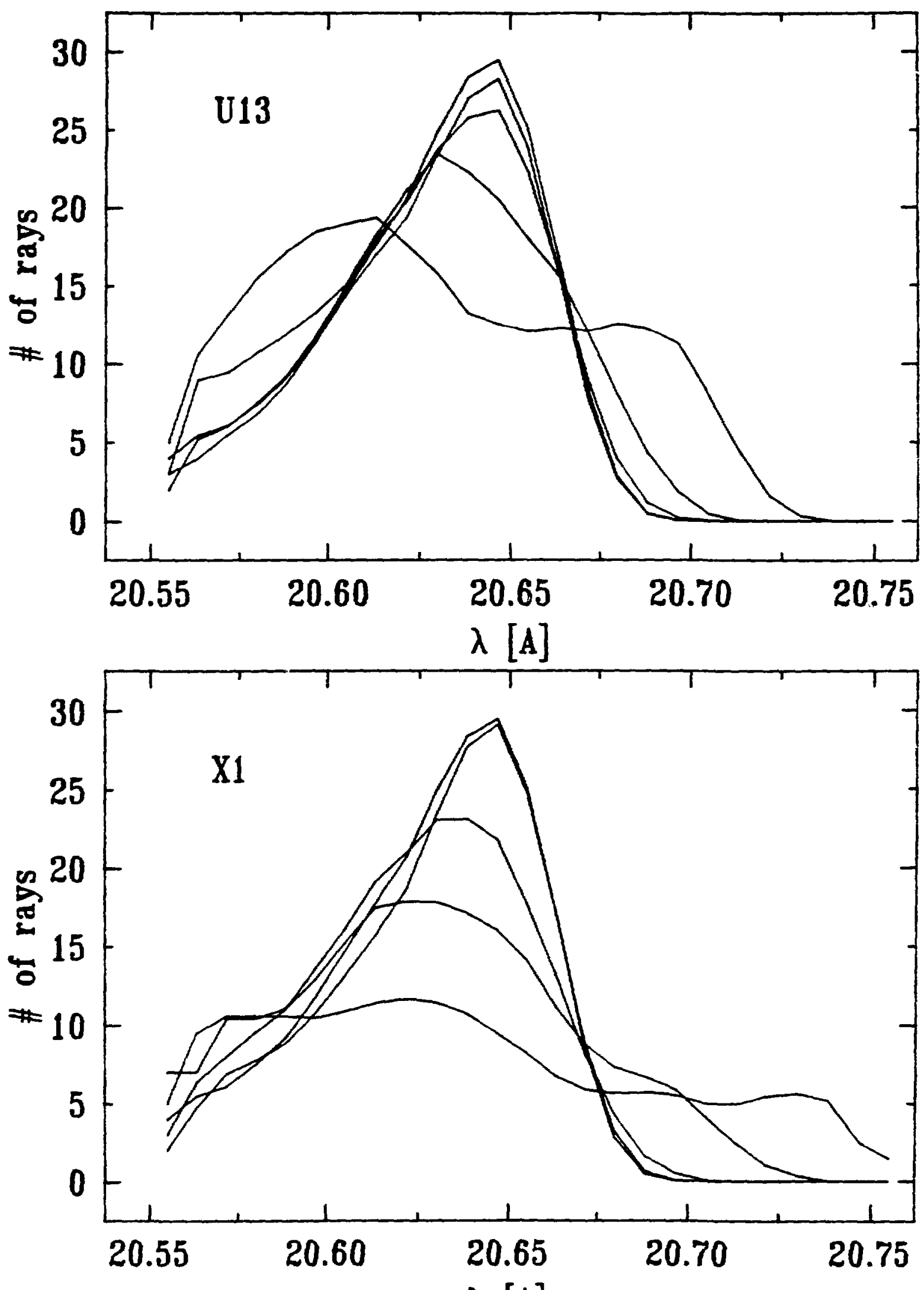


\section{Steven L. Hulbert}

Steven L. Hulber is an Associate Physicist in the National Synchrotron Light Source Department (NSLS) at Brookhaven National Laboratory. His interests are in the field of soft $x$-ray photoemission spectroscopy. He is in charge of the design, construction, commissioning, and use of the U13U high flux / high resolution branch line connected to the hybrid wiggler/undulator insertion device on the VUV electron storage ring at the NSLS. Dr. Hulber received his B.A. in Physics from Haverford College in 1976 and his Ph.D. in Physics from the University of Illinois in 1983. He was an Assistant Physicist in the Physics Department at Brookhaven National Laboratory from 1984 to 1986.

\section{Sushil Sharma}

Sushil Sharma is a Mechanical Engineer in the National Synchrotron Light Source Department (NSLS) at Brookhaven National Laboratory. He specializes in thermal deformation and stress in synchrotron radiation beamline components. Dr. Sharma received his Ph.D. in Theoretical and Applied Mechanics from University of Illinois ir: 1977. He was the group leader of the Tire Mechanics Division at GenCorp (Akron, Ohio) from 1977 to 1979. 


\section{DISCLAIMER}

This report was prepared as an acoount of work sponsored by an agency of the United States Government. Neither the United States Government nor any agency thereof, nor any of their employees, makes any warranty, express or implied, or assumes any legal liability or responsibility fer the accuracy, completeness, or usefulness of any information, apparatus, product, or process discinsed, or represents that its use would not infringe privately owned rights. Reference herein ic any specific commercial product, process, or service by trade name, trademark, manufacturs, or otherwise does not necessarily constitute or imply its endorsement, recommendation, or favoring by the United States Government or any agency thereof. The views and opinions of authors expressed herein do not necessarily state or reflect those of the United States Government or any agency thereof. 